\title{
Prácticas de prevención y control frente a la infección por Sars-Cov2 en la población
} peruana

\section{Prevention and control practices against Sars-Cov2 infection in the peruvian population}

Daniel Fernandez-Guzman ${ }^{1, \mathrm{a}}$, David R. Soriano-Moreno ${ }^{2, \mathrm{~b}}$, Fabricio Ccami-Bernal ${ }^{3, \mathrm{~b}}$, Cristhian Rojas-Miliano $0^{4, \mathrm{~b}}$, Lucero Sangster-Carrasc ${ }^{5, \mathrm{~b}}$, Enrique A. Hernandez-Bustamante ${ }^{6, \mathrm{~b}}$, Elvira G. https://doi.org/10.35434/rcmhnaaa.2021.14Sup1.1149

\section{RESUMEN}

Objetivo: Describir las prácticas de prevención y control para la infección por SARS-CoV2 en la población peruana. Material y Métodos: Estudio observacional de tipo descriptivo. Se evaluó una muestra no probabilística de adultos residentes en algún departamento de Perú. Las prácticas preventivas fueron evaluadas en personas sin antecedente de COVID-19 y las prácticas de control en personas que lo habían padecido. Resultados: Se evaluó un total de 3630 peruanos (edad media 25,4 \pm 9,5) de los cuales el 3231 no indicaban el antecedente de COVID-19 y 399 refirieron haberlo padecido. Las medidas de prevención y control que se realizaron a menudo o siempre con mayor frecuencia fueron el utilizar mascarilla al salir de casa $(97,9 \%$ vs 87,7$)$, cubrirse su nariz y boca al estornudar o toser $(95,4 \%$ vs $89,9 \%)$, guardar distancia de los demás en la calle $(91,4 \%$ vs $74,7 \%)$, realizar el lavado de manos con agua y jabón al volver a casa $(92,5 \%$ vs $88,7 \%)$, y desinfectar las superficies de objetos y lugares personales ( $82,6 \%$ vs $77,4 \%)$. El $22,1 \%$ y $83,7 \%$, el $59,7 \%$ y 80,2 , y el $8,0 \%$ y $16,8 \%$ consumieron algún tipo de medicamento, planta medicinal y dióxido de cloro para la prevención y control de la infección de COVID-19, respectivamente. Conclusiones: En general, menos del $50 \%$ de los participantes realizaron prácticas de prevención y control frente a la COVID-19 a menudo o siempre.

Palabras Clave: Infecciones por Coronavirus; COVID-19; prevención y control; Factores Socioeconómicos; pandemias; Perú (Fuente: DeCS-BIREME).
Zamora-Huaringa ${ }^{7, b}$, Abraham De-Los-Rios-Pinto ${ }^{1, b}$, Wendy Nieto-Gutierrez ${ }^{8, a}$
FILIACIÓN

- Escuela Profesional de Medicina Humana, Universidad Nacional de San Antonio Abad del Cusco, Cusco, Perú. Unidad de Investigación Clínica y Epidemiológica, Escuela de Medicina, Universidad Peruana Unión, Lima, Perú. Facultad de Medicina, Universidad Nacional de San Agustín de Arequipa, Arequipa, Perú.

Facultad de Medicina Humana, Universidad Nacional del Centro del Peru, Huancayo, Perù.

Escuela de Medicina, Universidad Peruana de Ciencias Aplicadas, Lima, Perú.

Sociedad Cientifica de Estudiantes de Medicina de la Universidad Nacional de Trujillo, Trujillo, Perú.

Facultad de Medicina Humana, Universidad Ricardo Palma, Lima, Perú.

Lima, Perú.

Médico general.

b. Estudiante de medicina humana.

ORCID

. Daniel Fernandez-Guzman / 0000-0002-9441-1067 6. EnriqueA. Hernandez-Bustamante / 0000-0002-1974-5200 David R. Soriano-Moreno / 0000-0002-3690-0014

Fabricio Ccami-Bernal /0000-0003-3172-2113

Cristhian Rojas-Miliano / 0000-0002-3364-4940

Lucero Sangster-Carrasco / $0000-0002-4073-052$

Elvira G. Zamora-Huaringa / 0000-0003-4208-0582

Abraham De-Los-Rios-Pinto / 0000-0001-6546-6870

\section{CORRESPONDENCIA}

Wendy Nieto-Gutierrez, Universidad San Ignacio de Loyola / Dirección: Av. la Fontana 550, La Molina, Lima, Perú. Teléfono: (+51) (01) 3171000

EMAIL

wendy nieto22@hotmail.com

\section{CRITERIOS DEAUTORÍA}

Daniel Fernandez-Guzmán: Conceptualización y metodología; investigación, recolección de datos - curaduría de datos y visualización, análisis formal, redacción - borrador original y escritura - revisión y edición.

David R. Soriano-Moreno: Recolección de datos - curaduría de datos, análisis formal y escritura - revisión y edición.

Fabricio Ccami-Bernal: Recolección de datos - curaduría de datos, análisis formal y escritura - revisión y edición. Cristhian Rojas-Miliano: Recolección de datos - curaduría de datos, análisis formal y escritura - revisión y edición.

Lucero Sangster-Carrasco: Recolección de datos, escritura - revisión y edición.

EnriqueA. Hernandez-Bustamante: Recolección de datos, escritura - revisión y edición.

Elvira G. Zamora-Huaringa: Recolección de datos, escritura - revisión y edición.

Abraham De-Los-Rios-Pinto: Recolección de datos, escritura - revisión y edición.

Wendy Nieto-Gutierrez: Metodología; administración del proyecto y supervisión; curaduría de datos, análisis formal, redacción - borrador original y escritura - revisión y edición.

CONFLICTOS DE INTERÉS

Los autores niegan conflictos de interés.

FINANCIAMIENTO

Autofinanciamiento.

AGRADECIMIENTOS

Se agradece a Randy Velasquez-Fernandez, Noelia Morocho-Alburqueque, Naomi Coba-Villan, Angelica Diaz-Corrale, Antony Pinedo-Soria, Valentina Murrieta-Ruiz, Pamela Grados-Espinoza, Carlos Quispe-Vicuña, Jherardy Lishner Raby Maryluz Castro-Quispe, Alejandra M Campos-Urbina, Ronald Apaza-Pillco y Fabián Andrés Chavéz-Ecos por su ayuda en la recolección de datos.

\section{REVISIÓN DE PARES}

Recibido: 25/07/2021

Aceptado: 27/09/2021

\section{COMO CITAR}

Fernandez-Guzman, D., Soriano-Moreno, D., Ccami-Bernal, F., Rojas-Miliano, C., Sangster-Carrasco, L., HernándezBustamante, E., Zamora-Huaringa, E., De-Los-Ríos-Pinto, A., \& Nieto-Gutierrez, W. (2021). Prácticas de prevención y control frente a la infección por Sars-Cov2 en la población peruana. Revista Del Cuerpo Médico Hospital Nacional
Almanzor Aguinaga Asenjo, 14(Sup1), 13 - 21. https://doi.org/10.35434/rcmhnaaa.2021.14Sup1.1149 


\section{ABSTRACT}

Objetive: To describe the prevention and control practices for the infection to SARS-COV2 in the Peruvian population. Material and Methods: Observational descriptive study. We evaluated a non-probabilistic sample of adult residents in some departments of Peru. Preventive practices were evaluated in people without a history of COVID-19 and control practices in people who had suffered it. Results: We evaluated 3630 Peruvians (mean age $25.4 \pm 9.5$ ), of that 3231 don't have a history of COVID-19 and 399 who had suffered it. The prevention and control practices that were realized often or always, with more frequencies, was the use of a mask when they go out home (97.9\% vs 87.7$)$, cover their nose or mouth when they sneeze $(95.4 \%$ vs $89.9 \%)$, save the distance to other people in the street $(91.4 \%$ vs $74.7 \%)$, wash their hands when they came home $(92.5 \%$ vs $88.7 \%)$, and disinfect the objects and personal places $(82.6 \%$ vs $77.4 \%)$. The $22.1 \%$ and $83.7 \%$, the $59.7 \%$ and 80.2 , and the $8.0 \%$ and $16.8 \%$ consumed some type of medicine, medicinal plant, and chlorine dioxide to prevent and control the infection, respectively. Conclusion: In general, less than $50 \%$ of the participants performed prevention and control practices against COVID-19 often or always.

Keywords: Coronavirus infections; COVID-19; pandemics: prevention and control; Socioeconomic Factors; Peru. (Source: DeCS-BIREME).

\section{INTRODUCCIÓN}

La pandemia por COVID-19 ha constituido un verdadero reto para los sistemas de salud de todo el mundo ${ }^{(1,2)}$, registrando a finales de diciembre de 2020, más de 100 millones de contagiados y más de 2 millones de defunciones a nivel mundial $l^{(3)}$.

Desde el comienzo de la pandemia, la Organización Mundial de la Salud (OMS) y el Centro para el Control y Prevención de Enfermedades (CDC), recomendaron emplear medidas de prevención y control de la infección ${ }^{(4,5)}$, enfatizando el lavado de manos, el uso de mascarilla y el distanciamiento social, al ser efectivas en pandemias pasadas contra virus respiratorios $^{(6)}$. Por lo que, diversos países alrededor del mundo decidieron incorporar dichas medidas dentro de sus normativas ${ }^{(7-9)}$. Siendo prioritaria su implementación en países de bajos y medianos ingresos, dado que sus sistemas de salud no estaban preparados para atender una cantidad masiva de casos de COVID-19 ${ }^{(10)}$.

El gobierno peruano, en su fin de contener la propagación del virus, impuso una inmovilización social, cierre de escuelas y paralización de actividades económicas como medidas iniciales para la contención del nuevo virus ${ }^{(6,11)}$. Asimismo, se exhortó a usar permanentemente la mascarilla, guardar distancia social, lavarse las manos frecuentemente y desinfectar artículos personales ${ }^{(12,13)}$. No obstante, la población peruana presentó una inadecuada adherencia a dichas medidas de prevención y control ${ }^{(10)}$, generando más de $1,107,239$ casos y 40,107 muertes para finales de enero ${ }^{(3)}$. En un panorama donde los casos siguen sumándose y la aparición de nuevas variantes del virus ${ }^{(3)}$ es necesario determinar las prevalencias de la prácticas de prevención y control de transmisión que se realizaron frente al virus para poder fortalecer e incentivar su continuación. El presente estudio con el objetivo del presente estudio fue describir las prácticas de prevención y control frente a la infección por SARS-COV2 en la población peruana.

\section{METODOLOGÍA}

\section{Diseño del estudio y población}

Realizamos un estudio observacional descriptivo. El cual evaluó una población peruana mayor de 18 años que residía en alguno de los 24 departamentos de Perú durante el 2020. Procedimientos.

Los participantes fueron enrolados mediante un muestreo no probabilístico tipo bola de nieve. Para lo que se utilizó una encuesta virtual utilizando la plataforma de Google forms, la cual estuvo habilitada por un periodo de 14 días (a partir del 7 de septiembre de 2020), tiempo en el que Perú se encontraba en pleno estado de emergencia y cuarentena focalizada ${ }^{(17)}$. La encuesta fue difundida mediante redes sociales (Facebook, WhatsApp, Instagram y Telegram) utilizando las cuentas personales de los autores y colaboradores que pertenecían a los 24 departamentos del Perú. Cada autor y colaborador difundió la encuesta en su respectivo departamento, solicitándose el llenado para con sus familiares y amistades. Además, se publicó la encuesta en grupos digitales representativos (universidades, localidades, barrios, etc.) y de libre acceso, que contuvieran población general peruana. Cuestionario.

Con base en el propósito de la investigación, los investigadores diseñaron el cuestionario tomando en cuenta estudios previos ${ }^{(18,19)}$, siendo evaluada y aprobada para su usó por tres médicos expertos en metodología de investigación. El cuestionario constó de tres secciones: 1) Características sociodemográficas personales y familiares; 2) perspectivas y prácticas de prevención de personas que no contaban con el antecedente de COVID-19 y 3) perspectivas y medidas realizadas para el control de la transmisión de las personas con el antecedente de COVID-19. Asimismo, se incluyó una presentación, el título, el objetivo del estudio, aspectos éticos que conllevaba, los criterios de inclusión, las instrucciones para el llenado, y una pregunta que determinaba el consentimiento informado, para posterior a ello, iniciar el cuestionario autoadministrado. 
La primera sección se usó para recolectar datos sobre las características sociodemográficas de los participantes como el género, edad, estado civil, grado de instrucción, vínculo laboral, lugar de residencia, seguro de salud, número de personas dentro del hogar y el estrato socioeconómico. Así mismo, se indago acerca de la presencia de algún estudiante o profesional de la salud dentro de la unidad familiar, la fuente de información de donde adquirió su conocimiento sobre el COVID-19 y si el encuestado o alguno de los miembros en su hogar presentaba alguna comorbilidad o si había padecido de COVID-19.

En la segunda y tercera sección se recopiló las prácticas y perspectivas de aquellas personas que no contaban con el antecedente de COVID-19 y que habían tenido la enfermedad, respectivamente.

La validez de fondo y contenido, fue confirmada por un panel de médicos; realizándose, además, el cálculo del Alpha de Cronbach, post aplicación del cuestionario. Resultando buena la validez de la encuesta global $(0,70)$, así como, cada uno de los factores: prácticas ( 25 ítems) y perspectivas (15 ítems), cuyo alfa fue 0,70 y de 0,59 , respectivamente.

Desenlaces: prácticas de prevención y control del COVID-19 Para el grupo de participantes sin antecedentes de COVID-19, se consideró 13 ítems para estimar las prácticas de prevención, mientras que para los participantes con antecedente de COVID-19 se evaluaron 7 ítems para evidenciar las prácticas de control frente a la transmisión del virus. Ambas comprobaban la frecuencia con que se realizaban algunas medidas recomendadas por el Ministerio de Salud del Perú ${ }^{(17,20)}$.

Para ambos desenlaces, se estimó la frecuencia de las medidas a través de la pregunta: "Usted ¿Con que frecuencia realiza...?", para los que no padecieron la infección, y "Usted durante su padecimiento ¿Con que frecuencia realizaba...?”, para los que, si la tuvieron, cuyas opciones de respuesta en ambos casos fueron codificadas por una escala tipo Likert que tomaba un valor de 0 "nunca", 1 "rara vez", 2 "a veces", 3 "a menudo" y 4 "siempre". Se calificó con un puntaje inverso por ser preguntas negativas a los ítems de llevar las manos sin lavar al rostro, salir acompañado para las prácticas preventivas y saludar entrando en contacto para las prácticas preventivas y de control.

Con respecto a la frecuencia de actividades realizadas con potencial de aumentar el riesgo de exposición al SARS-CoV-2 (salir de casa, ir a mercados o bancos, usar transporte público y acudir a actividades sociales) fue evaluado mediante una escala numérica que buscaba explorar el número de días en los que se expuso a dichas situaciones en las dos últimas semanas previas a la encuesta para el grupo sin antecedente de COVID-19 y en la última semana de enfermedad o aislamiento en el grupo que sí padeció la infección.

\section{Análisis estadístico}

El análisis de los datos fue realizado en el programa Stata versión 16.0. Se utilizaron frecuencias absolutas y relativas para la descripción de las variables categóricas, y medidas de
Tabla 1. Características de la población estudiada

\begin{tabular}{|c|c|c|c|}
\hline Variables & $\begin{array}{c}\text { Participantes sin } \\
\text { diagnóstico de COVID-19 } \\
\qquad(\mathrm{N}=3231)\end{array}$ & $\begin{array}{l}\text { Participantes con } \\
\text { diagnóstico de } \\
\text { COVID-19 } \\
(\mathrm{N}=399)\end{array}$ & $\begin{array}{c}\text { Total } \\
(\mathrm{N}=3630)\end{array}$ \\
\hline Edad $^{*}$ & $25.07 \pm 9.23$ & $28.00 \pm 11.23$ & $25.40 \pm 9.51$ \\
\hline \multicolumn{4}{|l|}{ Sexo } \\
\hline Masculino & $1204(37,26)$ & $163(40,85)$ & $1367(37,66)$ \\
\hline Femenino & $2027(62,74)$ & $236(59,15)$ & $2263(62,34)$ \\
\hline \multicolumn{4}{|l|}{ Estado civil } \\
\hline Soltero & $399(12,35)$ & $94(23,56)$ & $493(13,58)$ \\
\hline Casado o conviviente & $2832(87,65)$ & $305(76,44)$ & $3137(86,42)$ \\
\hline \multicolumn{4}{|l|}{ Grado de instrucción } \\
\hline Analfabeto & $8(0,25)$ & $6(1,50)$ & $14(0,39)$ \\
\hline Primaria & $4(0,12)$ & $3(0,75)$ & $7(0,19)$ \\
\hline Secundaria & $761(23,55)$ & $82(20,55)$ & $843(23,22)$ \\
\hline Superior & $2468(76,08)$ & $308(77,19)$ & $2766(76,19)$ \\
\hline \multicolumn{4}{|l|}{ Región } \\
\hline Costa & $1581(48,93)$ & $183(45,86)$ & $1764(48,60)$ \\
\hline Sierra & $1369(42,37)$ & $137(34,34)$ & $1506(41,49)$ \\
\hline Selva & $281(8,70)$ & $79(19,80)$ & $360(9,92)$ \\
\hline \multicolumn{4}{|l|}{ Zona de residencia } \\
\hline Urbana & $2741(84,83)$ & $330(82,71)$ & $3071(84,60)$ \\
\hline Rural & $490(15,17)$ & $69(17,29)$ & $559(15,40)$ \\
\hline \multicolumn{4}{|l|}{ Vínculo laboral } \\
\hline No & $2509(77,65)$ & $242(60,65)$ & $2751(75,79)$ \\
\hline Sí & $722(22,35)$ & $157(39,35)$ & $879(24,21)$ \\
\hline \multicolumn{4}{|l|}{ Seguro de salud } \\
\hline Ninguno & $974(30,15)$ & $102(25,56)$ & $1076(29,64)$ \\
\hline SIS & $1204(37,26)$ & $131(32,83)$ & $1335(36,78)$ \\
\hline EsSalud & $645(19,96)$ & $101(25,31)$ & $746(20,55)$ \\
\hline Otros & $408(12,63)$ & $65(16,29)$ & $473(13,03)$ \\
\hline \multicolumn{4}{|l|}{ Estrato socioeconómico } \\
\hline Estrato alto & $95(2,94)$ & $10(2,51)$ & $105(2,89)$ \\
\hline Estrato medio-alto & $1231(38,10)$ & $162(40,60)$ & $1393(38,37)$ \\
\hline Estrato medio-bajo & $1054(32,62)$ & $131(32,83)$ & $1185(32,64)$ \\
\hline Estrato obrero & $781(24,17)$ & $92(23,06)$ & $873(24,05)$ \\
\hline Estrato marginal & $70(2,17)$ & $4(1,00)$ & $74(2,04)$ \\
\hline \multicolumn{4}{|l|}{ Apoyo del gobierno } \\
\hline No & $2239(69,30)$ & $297(74,44)$ & $2536(69,86)$ \\
\hline Sí & $992(30,70)$ & $102(25,56)$ & $1094(30,14)$ \\
\hline \multicolumn{4}{|c|}{$\begin{array}{l}\text { Profesional de la salud dentro } \\
\text { de la unidad familiar }\end{array}$} \\
\hline No & $3021(93,50)$ & $351(87,97)$ & $3372(92,89)$ \\
\hline $\mathrm{Si}$ & $210(6,50)$ & $48(12,03)$ & $358(7,11)$ \\
\hline \multicolumn{4}{|c|}{$\begin{array}{l}\text { Estudiante de ciencias de la } \\
\text { salud dentro de la unidad } \\
\text { familiar }\end{array}$} \\
\hline No & $2645(81,86)$ & $342(85,71)$ & $2987(82,29)$ \\
\hline Sí & $586(18,14)$ & $57(14,29)$ & $643(17,71)$ \\
\hline \multicolumn{4}{|c|}{$\begin{array}{l}\text { Fuente donde adquiere } \\
\text { información sobre COVID-19 }\end{array}$} \\
\hline Redes sociales & $1098(33,98)$ & $128(32,08)$ & $1226(33,77)$ \\
\hline Medios de prensa & $1173(36,30)$ & $149(37,34)$ & $1322(36,42)$ \\
\hline Información medicas & $847(26,21)$ & $109(27,32)$ & $956(26,34)$ \\
\hline Amigos y familiares & $113(3,50)$ & $13(3,26)$ & $126(3,47)$ \\
\hline \multicolumn{4}{|c|}{ Comorbilidades para COVID-19 } \\
\hline No & $2757(85,33)$ & $306(76,69)$ & $3063(84,38)$ \\
\hline Sí & $474(14,67)$ & $93(23,31)$ & $567(15,62)$ \\
\hline \multicolumn{4}{|c|}{ Familiar con comorbilidad para } \\
\hline \multicolumn{4}{|c|}{ COVID-19 } \\
\hline No & $1587(49,12)$ & $181(45,36)$ & $1768(48,71)$ \\
\hline Sí & $1644(50,88)$ & $218(54,64)$ & $1862(51,29)$ \\
\hline \multicolumn{4}{|c|}{$\begin{array}{l}\text { Familiar con diagnóstico del } \\
\text { COVID-19 }\end{array}$} \\
\hline No & $2397(74,19)$ & $40(10,03)$ & $2437(67,13)$ \\
\hline Sí & $834(25,81)$ & $359(89,97)$ & $1193(32,87)$ \\
\hline \multicolumn{4}{|c|}{ Familiar fallecido por COVID-19 } \\
\hline No & $3188(98,67)$ & $372(93,23)$ & $3560(98,07)$ \\
\hline Sí & $43(1,33)$ & $27(6,77)$ & $70(1,93)$ \\
\hline
\end{tabular}

Media tesviación estánder 
tendencia central y dispersión para las variables numéricas. Las distribuciones de la frecuencia de actividades de riesgo de exposición al COVID-19 se trazaron mediante el método de densidades de Kernel.

\section{RESULTADOS}

Se encuestó un total de 3630 participantes (edad media $25,4 \pm 9,51,62,3 \%$ mujeres), de las cuales el $48,6 \%$ pertenecían a la región costa; $84,6 \%$ residían en una zona urbana; $76,2 \%$ tenía un grado de instrucción superior; $29,6 \%$ no se encontraban afiliados a ningún seguro de salud; $15,6 \%$ refirió tener por lo menos una comorbilidad; y $51,3 \%$ refirieron que al menos un miembro de su familia tenía alguna comorbilidad relacionada a un peor desenlace frente al COVID-19.

Del total de la población, 3231 refirieron no tener o haber tenido el diagnóstico de COVID-19 (edad media 25,1 \pm 9.2 años, $62,7 \%$ fueron mujeres) y 399 haber tenido la enfermedad (edad media 18,0 $\pm 11,2,59,2 \%$ mujeres). (Tabla $1)$.

Con respecto a las medidas de prevención de la enfermedad, un $27,8 \%$ de los encuestados las cumplieron a menudo o siempre. Asimismo, un $49,4 \%$ de los participantes salían a veces, rara vez o nunca acompañado a las calles $(2,42 \pm 1,10$ veces en las últimas dos semanas), mientras que un $47,6 \%$ a menudo o siempre usaba careta facial al salir de casa $(2,2 \pm 1,5$ veces en las últimas dos semanas). A cerca de las medidas de control de la enfermedad, el $13,5 \%$ las cumplieron a menudo o siempre y el $31,3 \%$ de los participantes usaba careta facial al salir de casa a menudo o siempre $(1,6 \pm 1,5$ veces en la última semana de la enfermedad). (Tabla 2 ).

En cuanto a medidas preventivas obligatorias en el Perú, el uso a menudo o siempre de mascarillas fue del 98,0\% (3 [rango 1-5] veces renovaron la mascarilla en las últimas dos semanas). Mientras que, como medida de control de la enfermedad, el $87,7 \%$ usaron la mascarilla a menudo o siempre (4 [rango 3-7] veces renovaron la mascarilla en las últimas dos semanas). (Tabla 2).

Por otro lado, se reportaron prácticas alternativas frente al COVID-19. Se halló que la prevalencia de consumo de medicamentos con intención de prevención o control del COVID-19 fue del $22,1 \%$ y $83,7 \%$, respectivamente, siendo los fármacos más destacados para ambos grupos el paracetamol, la ivermectina (tanto humana como veterinaria) y los macrólidos, y la principal fuente de información para el consumo fue la información médica (prevención: 50,7\% y control: $82,3 \%)$. La prevalencia de consumo de otras sustancias para prevenir o control la enfermedad fue del $59,7 \%$ y $80,2 \%$, en el caso del consumo de plantas, y $8,0 \%$ y $16,8 \%$, para el consumo dióxido de cloro, respectivamente. (Tabla 3).

Finalmente, en cuanto a plantas medicinales, la principal fuente de información para su consumo tanto para prevención y control de la enfermedad fueron otras fuentes que incluye los amigos, familiares, etc., con un $56,9 \%$ y $57,8 \%$ respectivamente. (Tabla 3 ).

Tabla 2. Prácticas de prevención y control frente la infección de COVID-19

\begin{tabular}{|c|c|c|c|c|c|c|}
\hline \multirow{2}{*}{ Prácticas } & \multicolumn{3}{|c|}{$\begin{array}{l}\text { Participantes sin antecedente } \\
\text { de COVID-19 } \\
(\mathrm{N}=3231)\end{array}$} & \multicolumn{3}{|c|}{$\begin{array}{l}\text { Participantes con } \\
\text { antecedente de COVID-19 } \\
\qquad(\mathrm{N}=399)\end{array}$} \\
\hline & $\begin{array}{l}\% \text { Nunca, rara } \\
\text { vez o a veces }\end{array}$ & $\begin{array}{l}\text { \% A menudo o } \\
\text { siempre }\end{array}$ & Promedio* & $\begin{array}{l}\% \text { Nunca, rara } \\
\text { vez o a veces }\end{array}$ & $\begin{array}{l}\text { \% A menudo o } \\
\text { siempre }\end{array}$ & Promedio* \\
\hline \multicolumn{7}{|l|}{ Uso de equipos de protección personal } \\
\hline Frecuencia de uso de mascarilla al salir de casa & $66(2,04)$ & $3165(97,96)$ & $3,85 \pm 0,47$ & $49(12,28)$ & $350(87,72)$ & $3,42 \pm 0,91$ \\
\hline Veces en la que renovó la mascarilla† & & & $3(1-5)$ & & & $4(3-7)$ \\
\hline Frecuencia con la que usa careta facial al salir de casa & $1694(52,43)$ & $1537(47,57)$ & $2,24 \pm 1,50$ & $274(68,67)$ & $125(31,33)$ & $1,60 \pm 1,47$ \\
\hline \multicolumn{7}{|l|}{ Medidas de prevención personal } \\
\hline Frecuencia con la que lleva sus manos sin lavar a los ojos, nariz o bocał & $1000(30,95)$ & $2231(69,05)$ & $2,84 \pm 1,16$ & - & - & - \\
\hline Frecuencia con la que cubre su nariz y boca al estornudar o toser & $148(4,58)$ & $3083(95,42)$ & $3,70 \pm 0,60$ & $40(10,03)$ & $359(89,97)$ & $3,57 \pm 0,87$ \\
\hline Frecuencia con la que guarda distancia de los demás en la calle (al menos 1 m) & $277(8,57)$ & $2954(91,43)$ & $3,49 \pm 0,71$ & $101(25,31)$ & $298(74,69)$ & $1,07 \pm 0,40$ \\
\hline Frecuencia con la que sale acompañado cuando va a la calleł & $1634(50,57)$ & $1597(49,43)$ & $2,42 \pm 1,10$ & - & - & - \\
\hline Frecuencia con la que realiza el lavado de manos con agua y jabón al volver a casa & $242(7,49)$ & $2989(92,51)$ & $3,65 \pm 0,68$ & $45(11,28)$ & $354(88,72)$ & $3,48 \pm 0,91$ \\
\hline Tiempo de lavado de manos al llegar a casa & & & $20(20-30)$ & & & $20(20-30)$ \\
\hline Frecuencia con la que da la mano, choca puño o codo con sus amigosł & $1408(43,58)$ & $1823(56,42)$ & $2,53 \pm 1,27$ & $65(16,29)$ & $334(83,71)$ & $3,09 \pm 1,35$ \\
\hline Frecuencia con la que desinfecta las superficies de objetos y lugares personales & $561(17,36)$ & $2670(82,64)$ & $3,32 \pm 0,90$ & $90(22,56)$ & $309(77,44)$ & $1,11 \pm 0,4$ \\
\hline Frecuencia con la que desinfecta su vestimenta al llegar a casa & $1069(33,09)$ & $2162(66,91)$ & $2,89 \pm 1,24$ & - & - & - \\
\hline Frecuencia con la que desinfecta su calzado al llegar a casa & $902(27,92)$ & $2329(72,08)$ & $3,03 \pm 1,25$ & - & - & - \\
\hline Frecuencia con la que desinfecta sus compras al llegar a casa & $596(18,45)$ & $2635(81,55)$ & $3,32 \pm 1,02$ & - & - & - \\
\hline Frecuencia con la que desinfecta su dinero al llegar a casa & $846(26,18)$ & $2385(73,82)$ & $3,08 \pm 1,21$ & - & - & - \\
\hline General & $2333(72,21)$ & $898(27,79)$ & $3,11 \pm 0,57$ & $345(86,47)$ & $54(13,53)$ & $1,76 \pm 0,68$ \\
\hline
\end{tabular}

*Media $\pm D E$ o Mediana (rango intercuartílico).

† Número de veces o dias durante las dos últimas semanas previa a la encuesta para los que no tuvieron

el antecedente de COVID-19 y durante la última semana de enfermedad para los que tuvieron COVID-19.

₹ Pregunta negativa (valor máximo a respuesta "0" nunca). 
Tabla 3. Productos usados con intención de prevención y control de la infección de COVID-19.

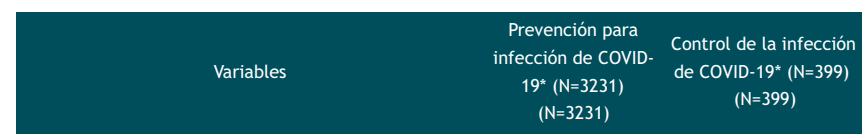

\section{Medicamentos}

Consumo

No

Veces que las usó en las dos últimas semanasł

Paracetamol

Ivermectina (de uso humano)

Macrólido (azitromicina o claritromicina)

Ivermectina (de uso veterinario)

AINES

Corticoides

Hidroxicloroquina

Heparina

Otros

Fuente de donde obtuvo información para el consumo

Redes sociales

Medios de prensa

Información médica

Otros (amigos, familiares, etc.)

Opinión sobre el consumo de ivermectina

Hace bien y no hace daño

Hace bien, pero hace poco daño

No hace bien, ni tampoco daño

No hace bien, pero si daño

No estoy informado

Opinión sobre el consumo de otros medicamentos

Hace bien y no hace daño

Hace bien, pero hace poco daño

No hace bien, ni tampoco daño

No hace bien, pero si daño

No estoy informado

Plantas medicinales

Consumo

No

Veces que las usó durante las dos últimas semanast

Tipo de plantas usadas

Eucalipto

Kion

Ajo

Matico

Otros (amigos, familiares, etc.)

Fuente de donde obtuvo la información para el consumo de pla

Redes sociales

Medios de prensa

Información medicas

Otros

Opinión sobre el consumo de plantas medicinales

$2517(77,40)$

$65(16,29)$

$714(22,10)$

$0,53 \pm 1,69 \quad 2,92 \pm 2,56$

$380(53,22) \quad 263(77,58)$

$329(46,08) \quad 198(58,41)$

$200(28,01) \quad 217(64,01)$

$135(18,91) \quad 48(14,16)$

$84(11,76) \quad 66(19,47)$

$54(7,56) \quad 94(27,73)$

$32(4,48) \quad 26(7,67)$

$14(1,96) \quad 23(6,78)$

$21(2,94) \quad 30(8,98)$

$73(10,22) \quad 9(2,69)$

$110(15,41) \quad 13(3,89)$

$362(50,7) \quad 275(82,34)$

$169(23,67) \quad 37(11,08)$

$465(14,39) \quad 40(10,03)$

$388(12,01) \quad 33(8,27)$

$695(21,51) \quad 104(26,07)$

$677(20,95) \quad 142(35,59)$

$1006(31,14) \quad 80(20,05)$

$518(16,03) \quad 33(8,27)$

$268(8,29) \quad 27(6,77)$

$366(11,33) \quad 86(21,55$

$215(6,65) \quad 90(22,56)$

$1864(57,69) \quad 163(40,85)$

$1303(40,33) \quad 79(19,80)$

$1928(59,67) \quad 320(80,20)$

$1,98 \pm 3,44 \quad 3,18 \pm 2,69$

$1498(77,70) \quad 281(87,81)$

$1286(66,70) \quad 213(66,56)$

$1027(53,27) \quad 193(60,31)$

$859(44,55) \quad 164(51,25)$

$223(69,69)$

$203(10,53) \quad 33(10,31)$

$296(15,35) \quad 38(11,88)$

$332(17,22) \quad 64(20.00)$

$1097(56,90) \quad 185(57,81)$

Hace bien, pero hace poco daño

No hace bien, ni tampoco daño

No hace bien, pero si daño

No estoy informado

Dióxido de cloro

Consumo

No

Veces en la que usó durante las dos últimas semanasł

Opinión sobre el consumo de dióxido de cloro

Hace bien y no hace daño

Hace bien, pero hace poco daño

No hace bien, ni tampoco daño

No hace bien, pero si daño

No estoy informado

$93(2,88)$

$729(22,56)$

$423(13,09)$

$1188(36,77)$

$798(24,70)$

$15(3,76)$

$54(13,53)$

$67(16,79$

$196(49,12$

$67(16,79)$

$2972(91,98) \quad 332(83,21)$

$259(8,02) \quad 67(16,79)$

$0,34 \pm 1,60 \quad 0,50 \pm 1,42$

$1538(47,60) \quad 163(40,85)$

$146(4,52) \quad 19(4,76)$

$258(7,99) \quad 32(8,02)$

$143(4,43) \quad 27(6,77)$

$1146(35,47)$

Se considero el consumo de sustancias en las dos últimas semanas previa a la encuesta para los que no tuvieron el antecedente de COVID-19 y durante la última semana de enfermedad para los que tuvieron COVID19. + Media $\pm D$

Con respecto a las actividades que aumentan la exposición al COVID-19, en el grupo que no tuvo COVID-19, se encontró que las personas que a menudo o siempre aplicaban las prácticas de prevención salieron una menor cantidad de veces de su casa para alguna actividad de riesgo (mediana 2 [1-5] vs 3 [27]) comparado con los que realizan casi nunca o siempre prácticas de prevención. Sin embargo, se observó un patrón similar en ambos grupos en las veces que salió para ir a los bancos o mercados (mediana 2 [1-3] vs 2 [1-4]), usar el transporte público (mediana 0 [0-2] vs 0 [0-2]), y participar de actividades sociales (mediana 0 [0-0] vs 0 [0-0]). (Figura 1 ) Por otro lado, en el grupo que tuvo COVID-19, se encontró que los encuestados reportaban una mediana de 0 veces de haber salido de casa durante la última semana de aislamiento o previo a su recuperación, ya sea para ir a los bancos o mercados usado el transporte público y haber participado de actividades sociales, tanto en los que realizaban las medidas de control menudo o siempre como los que casi nunca o nunca lo hacían. (Figura 1).

(A)
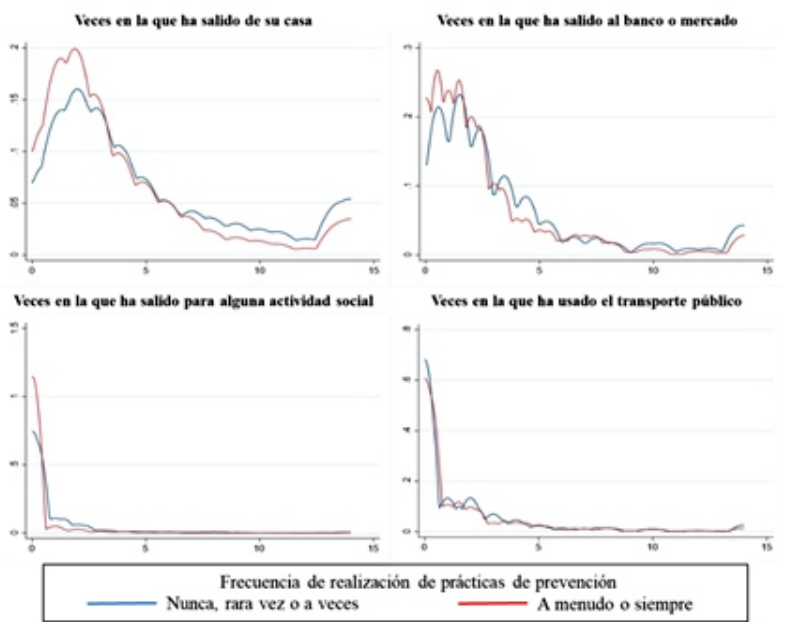

practicas de prevención

A menudo o siempre

(B)
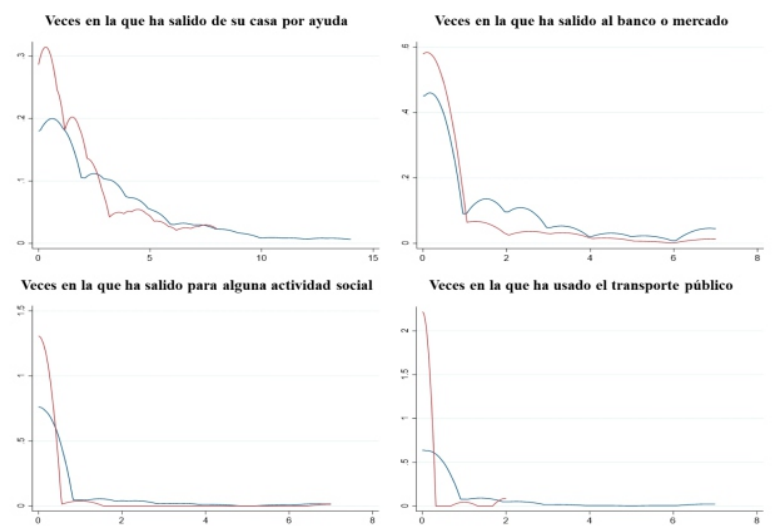

Frecuencia de re

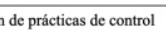

"Eje "X" densidades; eje "Y" frecuencia de veces que salió para realizar la actividad.

Figura 1.

Distribución de frecuencia de actividades de riesgo de exposición al SARS-CoV-2 según prácticas de prevención y control, por el método de densidades de Kernel. (A) Frecuencia de veces en la que ha salido en las últimas dos semanas de su casa, banco o mercado, actividad social y que ha utilizado el transporte público durante las dos últimas semanas. (B) Frecuencia de veces en la que ha salido en las últimas dos semanas de su casa y las veces en las que salió en la última semana al banco o mercado, actividad social, transporte público durante la última semana de enfermedad COVID-19. 


\section{DISCUSIÓN}

Nuestros resultados sugieren que tras seis meses desde el primer caso de COVID-19 en Perú(20) y aún durante la cuarentena focalizada y estado de emergencia vigentes ${ }^{(17)}$, la población peruana presentó una prevalencia baja de adherencia a las medidas de prevención y control frente al COVID-19. Este hecho es consistente con lo observado en la pandemia del 2009 de Influenza A (N1N1), donde las medidas impuestas fueron practicadas por una minoría de peruanos, explicando que este suceso posiblemente se dio por una falta de conocimientos sobre la enfermedad ${ }^{(23)}$. Esta pobre cultura de adherencia a las prácticas de prevención o control podría explicar el impacto que conllevó la pandemia del COVID-19 en la salud de los peruanos ${ }^{(24)}$, ubicándolo en la actualidad como el país sudamericano con mayor número de muertes por cada 100000 habitantes $^{(3)}$.

La población de estudio estuvo conformada por participantes con y sin antecedente de haber padecido COVID-19. División relevante para comprender la diferencia en las prácticas de ambos grupos, dado que son dos poblaciones que percibían la patogenicidad y letalidad del virus de forma diferente. Esta estratificación no ha sido evaluada en estudios similares previos $^{(25-28)}$, por lo que nuestros hallazgos, podrían ser punto de partida para futuras investigaciones que busquen explicar el impacto de las prácticas de control en las personas que pasaron la COVID-19 sobre la transmisión en la comunidad.

La presencia mayoritaria de jóvenes con grado de instrucción superior en ambos grupos, pudo deberse a la naturaleza del enrolamiento, lo que concuerda con estudios similares, cuyo tipo de muestreo también fue no probabilístico y donde se usó las redes sociales para la difusión de la encuesta (virtual autoadministrada) ${ }^{(25,26)}$. El tener una población predominantemente joven, debido al bajo riesgo de desarrollar enfermedad grave por COVID-19, puede conllevar a suponer una menor preocupación de contagiarse por parte de los participantes ${ }^{(29)}$. Pudiendo subestimar la frecuencia de realización de prácticas preventivas y de control en la población peruana. No obstante, al ser potenciales transmisores para el entorno familiar ${ }^{(30)}$, resulta beneficioso identificar las prácticas de prevención o control en este grupo etario como representantes del riesgo de transmisión que existe en los hogares peruanos.

La principal fuente de información usada, en nuestra población, para COVID-19 fueron los medios de prensa (televisivas, radios, etc.) y las redes sociales en ambos grupos de estudio, medios con los que los hogares peruanos estaban familiarizados previo a la pandemia ${ }^{(31)}$. No obstante, muchas de las noticias impartidas en estos medios no son del todo ciertas o no son respaldadas con evidencia científica de calidad $^{(16)}$, pudiendo conllevar a desplazar, inclusive, la realización de medidas de prevención y control recomendadas por organismos de salud.

Las medidas impartidas por el gobierno peruano han sido establecidas de forma temprana para promover y exigir la prevención de la infección del SARS-CoV-2 en la población, siendo algunas de carácter obligatorio como el uso de mascarillas ${ }^{(20)}$. A pesar de esto, se ha encontrado un porcentaje menor al $100 \%$ en el uso de mascarillas a menudo o siempre, siendo menor en el grupo que padeció COVID-19. Si bien la ejecución de esta medida es responsabilidad de las personas, en Perú, existe una falta de fiscalización del cumplimiento de las leyes, cuyo impacto puede ser relacionado en una desestimación de las normas nacionales en el ciudadano ${ }^{(17)}$.

Así mismo, la norma técnica peruana recomendó cambiar las mascarillas quirúrgicas en promedio cada 3 días $^{(3)}$. Si bien encontramos que el uso de la mascarilla para un posterior recambio variaba entre 1 a 5 días (mediana 3), para el grupo sin COVID-19, y de 3 a 7 (mediana 4), para los que padecieron la enfermedad, observamos que el recambio fue más largo en aquellos que tuvieron COVID-19, desencadenando un mayor riesgo de transmisión y contagio al entorno del individuo (32). La inadecuada frecuencia de recambio en ambos grupos, pudo deberse a la falta de recursos económicos en la población ${ }^{(17)}$, tomando en cuenta la afectación de la economía peruana y el incremento en los precios de mascarillas y desinfectantes durante la pandemia ${ }^{(33)}$.

El uso de la careta facial a menudo o frecuente fue menor al $50 \%$ en ambos grupos; esto pudo deberse a que su uso no fue recomendado de forma obligatoria por el estado peruano ${ }^{(20)}$, dado que es una medida cuya efectividad aún es controversial $^{(13)}$. Sin embargo, ciertas instituciones han impulsado su uso sobre todo en lugares con aglomeraciones de público (buses, oficinas, etc. ${ }^{(17)}$. Con respecto a otras medidas de protección personal, se encontró que más del $90 \%$ de peruanos sin COVID-19 cubrían su nariz y boca al estornudar y lavaban sus manos al regresar a casa a menudo o siempre, con una menor frecuencia en los que padecieron de la infección. A pesar de ello la prevalencia en ambos grupos fueron superiores a lo reportado durante pandemias pasadas $^{(23)}$, probablemente debido al mayor impacto de la pandemia del COVID-19 y la mayor presión por parte del estado para que dichas medidas se cumplan ${ }^{(20,24)}$. Sin embargo, se han observado resultados similares durante la actual pandemia en países como Vietnam ${ }^{(34)}$ y Arabia Saudita ${ }^{(19)}$.

Observamos que la mayor parte de encuestados guardaban el distanciamiento social a menudo o siempre, con una menor prevalencia entre aquellos que tuvieron COVID-19. Si bien cifras similares han sido reportadas en Irán (35), no se han encontrado estudios cuya evaluación de dichas variables fueron según el antecedente de COVID-19. Se conoce que el no realizar un distanciamiento social puede llevar a una mayor transmisión del virus hacia la gente sana, por la exposición de aerosoles inclusive utilizando mascarillas ${ }^{(36)}$, por lo que si bien se hace prioritario el enfoque de esta medida en aquellos que conocen que cuentan con la infección de COVID-19, también es imperativo que los que no tienen el diagnóstico lo incorporen en su rutina diaria para evitar contagio con casos no conocidos de COVID-19 o inclusive asintomáticos ${ }^{(37)}$.

Por otro lado, encontramos que casi la mitad de encuestados salen acompañados a la calle, hallazgo no evaluado en estudios similares ${ }^{(25-28)}$. Esto implica un mayor riesgo de exposición al virus para el resto del hogar, dado que posiblemente mantengan contacto entre ellos, y genera una mayor aglomeración en los lugares públicos, favoreciendo la transmisión del virus. Esto añadido a que más de la mitad de participantes (con y sin antecedente de COVID-19) 
mantienen un contacto físico a menudo o siempre con sus amigos, genera una alarma sobre la transmisibilidad de la infección. El evitar entrar en contacto con otras personas ha demostrado ser una medida efectiva para controlar la transmisión del virus ${ }^{(20)}$, disminuyendo un $82 \%$ de la probabilidad de infección ${ }^{(13)}$. Por lo que, es necesario recalcar y continuar promoviendo el evitar aglomeraciones, así como el contacto físico, dado que con las nuevas variantes ${ }^{(38)}$, una falta de implementación de estas, podría generar un aumento de casos y rebrotes futuros en la población peruana. Al igual que la mayoría de virus del tracto respiratorio, la transmisión del SARS-CoV-2 podría darse por fómites, puesto que el virus podría permanecer latente en las superficies de objetos entre horas a días, haciéndose necesario la desinfección de los mismos ${ }^{(39)}$. La mayoría de la población estudiada con y sin COVID-19 cumplía con las medidas de desinfección de sus objetos personales, en un porcentaje mayor a lo reportado en Etiopía ${ }^{(40)}$. Probablemente debido a que la desinfección de la vestimenta, el calzado y el dinero fue una de las medidas más recomendadas y promovidas por el Ministerio de Salud del Perú(41). No obstante, no contamos con la evidencia de la adecuada ejecución de estos procedimientos siguiendo estándares nacionales e internacionales, por lo que podrían haber participantes que la realizan pero con procedimientos inadecuados que no ayuden a la disminución del riesgo de infección por el nuevo coronavirus $^{(37)}$.

Asimismo, se observó una mayor frecuencia de realización de actividades de riesgo de exposición al COVID-19 en aquellos que, en promedio, realizaron prácticas de prevención nunca, raras veces o a veces. Esto es preocupante ya que la escasa realización de prácticas preventivas pone en un mayor riesgo de exposición al individuo para el contagio ${ }^{(42)}$. Mejores actitudes y niveles de conocimientos frente al COVID-19 se asocian a mejores prácticas ${ }^{(43)}$, por lo tanto, se debe llevar a cabo una estrategia de campaña de salud pública para aumentar el conocimiento a través de medios y estrategias efectivas a fin de ayudar a controlar la propagación de la enfermedad ${ }^{(18)}$.

El consumo de sustancias sin evidencia para la prevención y control de la infección por COVID-19 ha sido impulsada por diferentes líderes de opinión ${ }^{(44)}$, e inclusive el gobierno peruano $^{(45)}$. En este caso, observamos un consumo de medicamentos mayor del 50\% para el control de COVID-19, e igualmente, prevalencias altas de consumo de plantas medicinales para la prevención y control de la enfermedad. Sobre estos últimos, estudios realizados en otros países como Arabia Saudita ${ }^{(46)}$, África ${ }^{(47)}$ y Bangladés ${ }^{(48)}$, han reportado tendencias menores de consumo (del 10,2 a 57,6\%). Este elevado consumo de sustancias puede ser explicado de diferentes maneras. Principalmente puede ser producto del miedo generado en la población por enfermarse o presentar un desenlace grave de la enfermedad ${ }^{(49)}$. Sin embargo, también es necesario tomar en cuenta que este desenlace puede deberse a la propia cultura del país, como la costumbre del uso de la medicina alternativa ${ }^{(50)}$, así como, la costumbre de médicos y pacientes por brindar y tomar medicación aunque no fuera necesario ${ }^{(51)}$. En ambos casos, debido a que no se conoce el espectro de seguridad en la mayoría de estas sustancias y el beneficio es incierto, se debería recomendar no impulsar su consumo, pues además puede generar intoxicaciones ${ }^{(52,53)}$ o crear un sentimiento de falsa seguridad que conlleve a un descuido de la adherencia a prácticas preventivas y de controles comprobadas eficaces $^{(54)}$.

En general, ante la posibilidad de reinfección ${ }^{(55)}$, y la aparición de las nuevas variantes más transmisibles del SARSCoV- $2^{(38)}$, se recomienda continuar promoviendo e incentivando a la población a que continúe realizando las medidas de prevención o control independientemente haya o no tenido la enfermedad del COVID-19. Por lo que es necesario intensificar las estrategias de comunicación para difundir mensajes claves concretos acordes a la necesidad de cada público objetivo, encaminado a fortalecer, agrandar los conocimientos y rectificar las prácticas respecto a las medidas preventivas y de control para mitigar la transmisión del virus en la comunidad.

El presente estudio tiene ciertas limitaciones que hay que tener en cuenta. En primer lugar, se utilizó un muestreo no probabilístico para enrolar a los participantes, lo que dificulta la extrapolación de los resultados. Segundo, el hecho de que la encuesta fuera virtual y se distribuyera a través de las redes sociales, podría haber generado sesgo de selección. Finalmente, como la encuesta se basó en el autoinforme, la clasificación de los participantes se dio en base a sus respuestas. A pesar de estas limitaciones, este es un estudio que evaluó las prácticas preventivas contra la enfermedad COVID-19 en la población de las diferentes regiones del Perú. Asimismo, se realizó un análisis por subgrupos de si presentaban o no el antecedente de COVID-19 para conocer mejor las diferencias entre ambas situaciones. Por último, el tamaño de la muestra fue considerable, con un total de 3630 individuos.

En conclusión, menos del $50 \%$ de los participantes realizaron prácticas de prevención y control frente a la COVID-19 a menudo o siempre. Sin embargo, al evaluar por cada tipo de práctica, observamos que el usar la mascarilla al salir de casa, cubrirse su nariz y boca al estornudar o toser, guardar distancia de los demás en la calle, realizar el lavado de manos con agua y jabón al volver a casa, y desinfectar las superficies de objetos y lugares personales, fueron las realizadas con mayor frecuencia tanto para la prevención como el control de la enfermedad. Es necesario, la implementación de estrategias de educación y difusión de prácticas preventivas y control de COVID-19 por el gobierno e instituciones sanitarias, para concientizar a la población sobre las complicaciones de la infección por COVID-19 y los posibles desenlaces de esta.

\section{REFERENCIAS BIBLIOGRÁFICAS}

1. Rodriguez-Morales AJ, Sánchez-Duque JA, Hernández Botero $S$, Pérez-Díaz CE, Villamil-Gómez WE, Méndez CA, et al. Preparación y control de la enfermedad por coronavirus 2019 (COVID-19) en América Latina. Acta Medica Peru. 2020;37(1):3-7. doi:10.35663/amp.2020.371.909

2. Peeri NC, Shrestha N, Rahman MS, Zaki R, Tan Z, Bibi S, et al. The SARS, MERS and novel coronavirus (COVID-19) epidemics, the newest and biggest global health threats: what lessons have we learned? Int J Epidemiol. 2020;49(3):717-726. doi:10.1093/ije/dyaa033

3. Johns Hopkins Universiity. Mapa COVID-19 - Centro de recursos de coronavirus Johns Hopkins. COVID-19 Dashboard by the Center for Systems Science and Engineering (CSSE) at Johns Hopkins University. coronavirus.jhu.edu/map.html. Published 2020. Accessed March 3, 2021. 
4. World Health Organization. Rational use of personal protective equipment (PPE) for coronavirus disease (COVID-19) [Internet]. WHO Headquarters (HQ), WHO Worldwide; 2020 [cited 2020 dec 2]. Available from: https://bit.ly/2XR1Mvf

5. Centers for Disease Control and Prevention. How to Protect Yourself and Others; 2020 [cited 2020 dec 20]. Available from: https: / / bit.ly/3CD1K8W

6. World Health Organization. Recommendations to Member States to Improve Hand Hygiene Practices to Help Prevent the Transmission of the COVID-19 Virus; 2020 [cited 2020 dec 6]. Available from: https: / / bit.ly/3lFMeSU

7. Nussbaumer-Streit B, Mayr V, Dobrescu Al, Chapman A, Persad E, Klerings I, et al. Quarantine alone or in combination with other public health measures to control COVID-19: a rapid review. Cochrane dat aba se Syst Rev. $2020 ; 4$ (4): C D 013574 . doi:10.1002/14651858.CD013574

8. Wilder-Smith A, Freedman DO. Isolation, quarantine, social distancing and community containment: pivotal role for old-style public health measures in the novel coronavirus (2019-nCoV) outbreak. J Travel Med. 2020;27(2):1-4. doi:10.1093/jtm/taaa020

9. Saglietto A, D'Ascenzo F, Zoccai GB, De Ferrari GM. COVID-19 in Europe: the Italian lesson. Lancet. 2020;395(10230):1110-1111. doi:10.1016/S0140-6736(20)30690-5

10. Maxmen A. How poorer countries are scrambling to prevent a coronavirus disaster. Nature. 2020;580(7802):173-174. doi:10.1038/d41586-020-00983-9

11. Fernandez-Guzman D, Sangster-Carrasco L, Pinedo-Soria A. COVID-19 in Latin America and the Caribbean: what is known about the status of school reopening? J Public Health. December 2020:1-3. doi:10.1093/pubmed/fdaa205

12. Liang M, Gao L, Cheng C, Zhou Q, Uy JP, Heiner K, et al. Efficacy of face mask in preventing respiratory virus transmission: A systematic review and meta-analysis. Travel Med Infect Dis. 2020;36(3):101751. doi:10.1016/j.tmaid.2020.101751

13. Chu DK, Akl EA, Duda S, Solo K, Yaacoub S, Schünemann HJ, et al. Physical distancing, face masks, and eye protection to prevent person-to-person transmission of SARS-CoV-2 and COVID-19: a systematic review and meta-analysis. Lancet. 2020;395(10242):1973-1987. doi:10.1016/S0140-6736(20)31142-9

14. Rahmani AM, Mirmahaleh SYH. Coronavirus disease (COVID-19) prevention and treatment methods and effective parameters: A systematic literature review. Sustain Cities Soc. 2021;64:102568. doi:10.1016/j.scs.2020.102568

15. Martinez-Rivera RN, Taype-Rondan A. Overmedication in COVID-19 Context: A Report From Peru. J Clin Pharmacol. 2020;60(9):11551156. doi: 10.1002/jcph.1704

16. Alvarez-Risco A, Mejia CR, Delgado-Zegarra J, Del-Aguila-Arcentales $\mathrm{S}$, Arce-Esquivel AA, Valladares-Garrido MJ, et al. The Peru Approach against the COVID-19 Infodemic: Insights and Strategies. Am J Trop Med Hyg. 2020;103(2):583-586. doi:10.4269/ajtmh.20-0536

17. República del Perú. Decreto Supremo que modifica el Decreto Supremo $N^{\circ}$ 116-2020-PCM, Decreto Supremo que establece las medidas que debe observar la ciudadanía en la Nueva Convivencia Social y prorroga el Estado de Emergencia Nacional por las graves circunstancias que afectan la vida de la Nación a consecuencia del COVID-19. Perú: Presidencia del Consejo de Ministros; 2020 [citado el 10 de diciembre de 2020]. Disponible en: https: / / bit.ly/3Ey6Ipo.

18. Puspitasari IM, Yusuf L, Sinuraya RK, Abdulah R, Koyama H. Knowledge, Attitude, and Practice During the COVID-19 Pandemic: A Review. J Multidiscip Healthc. 2020; 13:727-733. doi:10.2147/JMDH.S265527

19. Al-Hanawi MK, Angawi K, Alshareef N, Qattan AMN, Helmy HZ, Abudawood Y, et al. Knowledge, Attitude and Practice Toward COVID19 Among the Public in the Kingdom of Saudi Arabia: A Cross-Sectional Study. Front Public Heal. 2020;8 (217):1-10. doi:10.3389/fpubh.2020.00217

20. Ministerio de Salud. Alerta Epidemiológica $N^{\circ} 12$ Ante El Incremento de Casos de COVID-19 En El Perú. Lima: Dirección General de Epidemiologia, MINSA; 2020 [citado el 10 de diciembre de 2020]. Disponible en: https: / / bit.ly/3hRvlDF

21. World Medical Association. World Medical Association Declaration of Helsinki. JAMA. 2013;310(20):2191. doi:10.1001/jama.2013.281053

22. Decreto Supremo que declara Estado de Emergencia Nacional por las graves circunstancias que afectan la vida de la Nación a consecuencia del brote del COVID-19-DECRETO SUPREMO-N ${ }^{\circ}$ 044-2020-PCM. Lima: Presidencia del Consejo de Ministros; 2020 [citado el 15 de diciembre de 2020]. Disponible en: https: / / bit.ly/2W3hQcA

23. Ávila Jeannette, Munayco César V., Gomez Jorge, Nunura Juan, Canahuiri Jerónimo. Conocimientos y prácticas sobre la nueva influenza A (H1N1) en trabajadores de salud y pacientes ambulatorios, Perú (mayo 2009). Rev. perú. med. exp. salud publica [Internet]. 2009 [citado 2021 Mar 2]; 26( 3 ): 328-332. Disponible en: https://bit.ly/3lK6oLD.

24. The Lancet. COVID-19 in Latin America: a humanitarian crisis. Lancet. 2020;396(10261):1463. doi:10.1016/S0140-6736(20)32328-X

25. Salman M, Mustafa ZU, Asif N, Zaidi HA, Hussain K, Shehzadi N, et al. Knowledge, attitude and preventive practices related to COVID-19: a cross-sectional study in two Pakistani university populations. Drugs Ther Perspect. 2020;36(7):319-325. doi:10.1007/s40267-020-007377

26. Gupta P, Gupta A, Dixit S, Kumar H. Knowledge, attitude, and practices regarding COVID-19: A cross-sectional study among rural population in a northern Indian District. J Fam Med Prim Care. 2020;9(9):4769. doi:10.4103/jfmpc.jfmpc 120620

27. Zhong B, Luo W, Li H-M, Zhang Q, Liu X, Li W-T, et al. Knowledge, attitudes, and practices towards COVID-19 among Chinese residents during the rapid rise period of the COVID-19 outbreak: a quick online cross-sectional survey. Int J Biol Sci. 2020;16(10):1745-1752. doi:10.7150/ijbs. 45221

28. Arp NL, Nguyen TH, Linck EJG, Feeney AK, Schrope JH, Ruedinger KL, et al. Use of face coverings in public during the COVID-19 pandemic: a $n$ observational study. medRxiv. 2020 doi:10.1101/2020.06.09.20126946

29. Bruine de Bruin W. Age Differences in COVID-19 Risk Perceptions and Mental Health: Evidence From a National U.S. Survey Conducted in March 2020. Journals Gerontol Ser B. 2021;76(2):e24-e26. doi:10.1093/geronb/gbaa074

30. Nikolai LA, Meyer CG, Kremsner PG, Velavan TP. Asymptomatic SARS Coronavirus 2 infection: Invisible yet invincible. Int J Infect Dis. 2020;100(2020):112-116. doi:10.1016/j.ijid.2020.08.076

31. Instituto Nacional de Estadística e Informática. Características de las viviendas, hogares y población - Encuesta Demográfica y Salud Familiar (ENDES 2019). Lima, INEI; 2019 [citado el 18 de diciembre de 2020]. Disponible en: https://bit.ly/3tYg0X3.

32. Wiersinga WJ, Rhodes A, Cheng AC, Peacock SJ, Prescott HC. Pathophysiology, Transmission, Diagnosis, and Treatment of Coronavirus Disease 2019 (COVID-19). JAMA. 2020;324(8):782. doi:10.1001/jama.2020.12839

33. Perú Retail. Coronavirus: Farmacias incrementan precios de alcohol y mascarillas. Lima, PERURETAIL; 2020 [citado el 12 de febrero de 2021]. Disponible en: https: / / bit.ly/3EPZhKy

34. Van-Nhu H, Tuyet-Hanh TT, Van NTA, Linh TNQ, Tien TQ. Knowledge, Attitudes, and Practices of the Vietnamese as Key Factors in Controlling COVID-19. J Community Health. 2020;45(6):1263-1269. doi:10.1007/s10900-020-00919-4

35. Honarvar B, Lankarani KB, Kharmandar A, Shaygani F, Zahedroozgar $M$, Rahmanian Haghighi MR, et al. Knowledge, attitudes, risk perceptions, and practices of adults toward COVID-19: a population and field-based study from Iran. Int J Public Health. 2020;65(6):731739. doi:10.1007/s00038-020-01406-2

36. Meyerowitz EA, Richterman A, Gandhi RT, Sax PE. Transmission of SARS-CoV-2: A Review of Viral, Host, and Environmental Factors. Ann Intern Med. 2021;174(1):69-79. doi:10.7326/M20-5008

37. Orenes-Piñero E, Baño F, Navas-Carrillo D, Moreno-Docón A, Marín JM, Misiego R, et al. Evidences of SARS-CoV-2 virus air transmission indoors using several untouched surfaces: A pilot study. Sci Total Environ. 2021;751:142317. doi:10.1016/j.scitotenv.2020.142317

38. Kidd M, Richter A, Best A, Cumley N, Mirza J, Percival B, et al. Svariant SARS-CoV-2 lineage B1.1.7 is associated with significantly higher viral loads in samples tested by ThermoFisher TaqPath RTqPCR. J Infect Dis. 2021. doi:10.1093/infdis/jiab082

39. Marquès M, Domingo JL. Contamination of inert surfaces by SARS CoV-2: Persistence, stability and infectivity. A review. Environ Res. 2021;193:110559. doi:10.1016/j.envres.2020.110559

40. Bante A, Mersha A, Tesfaye A, Tsegaye B, Shibiru S, Ayele G, et al. Adherence with COVID-19 Preventive Measures and Associated Factors Among Residents of Dirashe District, Southern Ethiopia. Patient Prefer Adherence. 2021; 15:237-249. doi:10.2147/PPA.S293647

41. El Peruano. Decreto Supremo que declara Estado de Emergencia Nacional por las graves circunstancias que afectan la vida de la Nación a consecuencia del brote del COVID-19-DECRETO SUPREMO-N 
044-2020-PCM. Lima, presidente del consejo de ministros; 2020 [citado 30 de noviembre de 2020]. Disponible en: https://bit.ly/3Aztk6K

42. Girum T, Lentiro K, Geremew M, Migora B, Shewamare S. Global strategies and effectiveness for COVID-19 prevention through contact tracing, screening, quarantine, and isolation: a systematic review. Trop Med Health. 2020;48(1):91. doi:10.1186/s41182-020-00285-w

43. Fang Y, Liu P, Gao Q. Assessment of Knowledge, Attitude, and Practice Toward COVID-19 in China: An Online Cross-Sectional Survey. Am J Trop Med Hyg. 2021;104(4):1461-1471. doi:10.4269/ajtmh.20-0452

44. Mostajo-radji MA. Pseudoscience in the times of crisis: How and why chlorine dioxide consumption became popular in Latin America during the COVID-19 pandemic (Preprint). 2020. Available from: https://osf.io/preprints/socarxiv/u9ehf/

45. Resolución Ministerial N 193-2020-MINSA | Gobierno del Perú; 2020 [citado el 20 de enero de 2021]. Disponible en: https: //bit.ly/3nWu3eM

46. Alyami HS, Orabi MAA, Aldhabbah FM, Alturki HN, Aburas WI, Alfayez Al, et al. Knowledge about COVID-19 and beliefs about and use of herbal products during the COVID-19 pandemic: A cross-sectional study in Saudi Arabia. Saudi Pharm J. 2020;28(11):1326-1332. doi:10.1016/j.jsps.2020.08.023

47. Sadio AJ, Gbeasor-Komlanvi FA, Konu RY, Bakoubayi AW, Tchankoni MK, Bitty-Anderson AM, et al. Assessment of self-medication practices in the context of the COVID-19 outbreak in Togo. BMC Public Health. 2021;21(1):58. doi:10.1186/s12889-020-10145-1

48. Ahmed I, Hasan M, Akter R, Sarkar BK, Rahman M, Sarker MS, et al. Behavioral preventive measures and the use of medicines and herbal products among the public in response to Covid-19 in Bangladesh: $\mathrm{A}$ cross-sectional study. Ghose B, ed. PLoS One. 2020;15(12):e0243706. doi:10.1371/journal.pone.0243706
49. Taquet M, Luciano S, Geddes JR, Harrison PJ. Bidirectional associations between COVID-19 and psychiatric disorder: retrospective cohort studies of 62354 COVID-19 cases in the USA. The Lancet Psychiatry. 2021;8(2):130-140. doi:10.1016/S22150366(20)30462-4

50. Mostacero-León J, López-Medina SE, De La Cruz-Castillo AJ, GilRivero AE, Alva Calderón R, Charcape Ravelo M. "Cold plants" and "Hot plants" potential resources in the prevention and / or treatment of COVID-19. Manglar. 2020;17(3):209-220. doi:10.17268/manglar.2020.031

51. Ecker L, Ruiz J, Vargas M, Del Valle LJ, Ochoa TJ. Prevalencia de compra sin receta y recomendación de antibióticos para niños menores de 5 años en farmacias privadas de zonas periurbanas en Lima, Perú. Rev Peru Med Exp Salud Publica. 2016;33(2):215. doi:10.17843/rpmesp.2016.332.2152

52. Kwok KO, Lai F, Wei WI, Wong SYS, Tang JWT. Herd immunity estimating the level required to halt the COVID-19 epidemics in affected countries. J Infect. 2020;80(6):e32-e33. doi:10.1016/j.jinf.2020.03.027

53. Román BR, Moscoso S, Chung SA, Terceros BL, Álvarez-Risco A, Yáñez JA. Treatment of COVID-19 in peru and bolivia, and self-medication risks. Rev Cuba Farm[Internet]. 2020 [cited 2020 Jan 22];53(2):1-20. Available from: https://hdl.handle.net/20.500.12724/11641

54. Montastruc J-L, Bondon-Guitton E, Abadie D, Lacroix I, Berreni A, Pugnet $\mathrm{G}$, et al. Pharmacovigilance, risks and adverse effects of selfmedication. Therapies. 2016;71(2):257-262. doi:10.1016/j.therap.2016.02.012

55. Mulder M, van der Vegt DSJM, Oude Munnink BB, GeurtsvanKessel CH, van de Bovenkamp J, Sikkema RS, et al. Reinfection of Severe Acute Respiratory Syndrome Coronavirus 2 in an Immunocompromised Patient: A Case Report. Clin Infect Dis. 2020;0(0). 